\title{
Eosinophil Activation: How the Cytoskeleton Shapes Immune Response
}

Joshua Mitchell and Deane Mosher

University of Wisconsin-Madison, Madison, Wisconsin, United States

Eosinophils (EOS) play an essential role in human health and disease. EOS are granule-containing leukocytes that contribute uniquely to the immune response through degranulation and release of granular proteins. Eosinophils are regularly associated with parasitic infection, but also respond to a broad range of stimuli, from bacterial infection to environmental antigens and mechanical injury. Disequilibrium in their activity can result in various clinical presentations such as eosinophilic asthma, esophagitis, and hyper-eosinophilic syndrome. However, eosinophils have remained understudied compared to other blood leukocytes. There is a critical need to understand the fundamental molecular mechanisms by which EOS become activated, which can result in significant downstream effects in human health.

Activation of EOS with interleukin-5 (IL5) and IL33 results in dramatic morphological and cytoskeletal rearrangements. The EOS becomes polarized, transitioning from a spherical to an acorn-like shape with distinct cellular domains. A uropod at the rear of the cell frames the nucleus, called the nucleopod, which serves as the primary signaling center of the activated EOS $^{1}$. The front of the cell has a ruffled migratory edge which is adjacent to the granulomere, which occupies the majority of the EOS. Upon activation, microtubules, actin, and vimentin undergo very distinct reorganization revealed by confocal and superresolution microscopy. The web-like vimentin network of naïve EOS condenses into the nucleopod, supporting the uropod-like extension that trails behind the cell. Microtubules span the nucleus to the apical tip of the nucleopod, connecting the granulomere to signal center of the activated EOS. Actin localizes to the leading edge of the EOS as well as the granulomere, providing the necessary motility for migration and granule anchoring in the body of the cell. Our investigation of the cytoskeletal response to EOS activation will help illuminate the fundamental molecular mechanisms that govern EOS polarization, from adhesion and migration to primary function within the immune system.

References

Han, S.-T. \& Mosher, D. F. IL-5 Induces Suspended Eosinophils to Undergo Unique Global Reorganization Associated with Priming. Am. J. Respir. Cell Mol. Biol. 50, 654-664 (2013). 